\title{
Oblivious Transfer Is Symmetric
}

\author{
Stefan Wolf and Jürg Wullschleger \\ Computer Science Department, ETH Zürich, Switzerland \\ $\{$ wolf, wjuerg\}@inf.ethz.ch
}

\begin{abstract}
We show that oblivious transfer of bits from $A$ to $B$ can be obtained from a single instance of the same primitive from $B$ to $A$. Our reduction is perfect and shows that oblivious transfer is in fact a symmetric functionality. This solves an open problem posed by Crépeau and Sántha in 1991.
\end{abstract}

\section{Introduction}

Modern cryptography is an increasingly broad discipline and deals with many subjects besides the classical tasks of encryption or authentication. An example is multi-party computation, where two or more parties, mutually distrusting each other, want to collaborate in a secure way in order to achieve a common goal, for instance, to carry out an electronic election. An example of a specific multi-party computation is secure function evaluation, where every party holds an input to a function, and the output should be computed in a way such that no party has to reveal unnecessary information about her input.

A primitive of particular importance in the context of two- and multi-party computation is oblivious transfer. In classical Rabin oblivious transfer [19] or Rabin OT for short, one of the parties - the sender - sends a bit $b$ which reaches the receiver with probability $1 / 2$; the sender hereby remains ignorant of about whether the message has arrived or not. In other words, Rabin OT is nothing else than a binary erasure channel. Another variant of oblivious transfer is chosen one-out-of-two oblivious transfer- $\left(\begin{array}{l}2 \\ 1\end{array}\right)$-OT for short- , where the sender sends two bits $b_{0}$ and $b_{1}$ and the receiver's input is a choice bit $c$; the latter then learns $b_{c}$ but gets no information about the other bit $b_{1-c}$. Chosen one-out-oftwo oblivious transfer can be generalized to a primitive where the sender sends $n$ messages, $k$ of which the receiver can choose to read: chosen $k$-out-of-n $l$-bit string oblivious transfer or $\left(\begin{array}{l}n \\ k\end{array}\right)-\mathrm{OT}^{l}$. One reason for the importance of oblivious transfer is its universality, i.e., it allows, in principle, for carrying out any twoparty computation [14].

Besides computational cryptographic security, which is based on the assumed hardness of certain computational problems and a limitation on the adversary's computing power, there also exists unconditional security, which is based on the fact that the information the potential adversary obtains is limited. This latter type of security withstands attacks even by a computationally unlimited adversary; clearly, it is, a priori, more desirable to realize cryptographic primitives in such an unconditionally secure way. Unfortunately, oblivious transfer is impossible to achieve in an unconditionally secure way from scratch, i.e., between

The original version of this chapter was revised: The copyright line was incorrect. This has been corrected. The Erratum to this chapter is available at DOI: 10.1007/978-3-540-34547-3_36

S. Vaudenay (Ed.): EUROCRYPT 2006, LNCS 4004, pp. 222-232, 2006.

(C) Springer-Verlag Berlin Heidelberg 2006 
parties connected by a noiseless channel; in fact, not even if this is a quantum channel over which the parties can exchange not only "classical" bits but quantum states [15]. However, if some additional weak and realistic primitives are available such as noisy channels and noisy correlations, then unconditional security can be often achieved [7], [6], [8], [13], [22], [23].

Another way of realizing unconditionally secure oblivious transfer is from (a weaker form of) oblivious transfer itself: All the variants of oblivious transfer have been shown equivalent to different extents. For instance, $\left(\begin{array}{l}2 \\ 1\end{array}\right)$-OT can be reduced to $m$ realizations of Rabin OT as long as a failure probability of $2^{-m}$ can be accepted [5]. On the other hand, $\left(\begin{array}{l}2 \\ 1\end{array}\right)-\mathrm{OT}^{l}$ can be reduced to $\Theta(l)$ realizations of $\left(\begin{array}{l}2 \\ 1\end{array}\right)$-OT-with or without failure probability, where the reduction can be made more efficient in terms of the hidden constant if a small probability of failure can be accepted [4]. In [2], a protocol was presented that reduces $\left(\begin{array}{l}2 \\ 1\end{array}\right)$-OT to a $\left(\begin{array}{l}2 \\ 1\end{array}\right)$-OT being available at an earlier point in time. This means that $\left(\begin{array}{l}2 \\ 1\end{array}\right)-\mathrm{OT}$ can be precomputed (or stored and used at any time later).

In [18] and [9], methods were proposed for obtaining $\left(\begin{array}{l}2 \\ 1\end{array}\right)-\mathrm{OT}$ from $A$ to $B$ from $n$ instances of $\left(\begin{array}{l}2 \\ 1\end{array}\right)$-OT from $B$ to $A$, where a failure probability of $2^{-\Theta(n)}$ has to be tolerated. The protocol of [18] is based on the realization of so-called "XOT" (i.e., the receiver can also choose to receive the XOR of the two bits sent) from two realizations of $\left(\begin{array}{l}2 \\ 1\end{array}\right)$ - TO - the reversed version of $\left(\begin{array}{l}2 \\ 1\end{array}\right)$-OT. Note, however, that the resulting reduction of $\left(\begin{array}{l}2 \\ 1\end{array}\right)-\mathrm{OT}$ to $\left(\begin{array}{l}2 \\ 1\end{array}\right)$ - TO of [18] also requires $\Theta(\log (1 / \varepsilon))$ realizations of $\left(\begin{array}{l}2 \\ 1\end{array}\right)$-TO if $\varepsilon$ is the tolerated failure probability.

\subsection{Our Contribution}

In [9], Crépeau and Sántha raised the question of whether it is possible to implement oblivious transfer in one direction using fewer instances of oblivious transfer in the other. In this paper, we answer this question with yes by presenting a protocol that needs one instance of oblivious transfer, one bit of communication and one bit of additional (local) randomness. All these parameters are optimal. Our reduction is very simple; in other words, the reversed version of oblivious transfer is basically just another way of looking at it. The symmetry is already there, oblivious transfer is symmetric.

Our reduction can be used to transform any protocol for $\left(\begin{array}{l}2 \\ 1\end{array}\right)$-OT-offering either computational or information-theoretic security for $A$ and $B$, respectively - into a protocol for oblivious transfer from $B$ to $A$ having exactly the same security both for $A$ and $B$ as the original protocol; no additional failure can occur.

\subsection{Outline}

In Section 3, we first present protocols from [2] that allow $\left(\begin{array}{l}2 \\ 1\end{array}\right)$-OT to be "stored", i.e., to transform oblivious transfer into an oblivious key. Then, we will show that such an oblivious key can very easily be reversed-by a simple XOR executed by both players on their local data. It follows that oblivious transfer can be reversed equally easily. In Section 4 we present an even simpler protocol for reversing oblivious transfer and prove its security. 


\section{Definitions and Security}

We define $\left(\begin{array}{l}2 \\ 1\end{array}\right)$-OT as a black-box (see Figure 1 ).

Definition 1. By $\left(\begin{array}{l}2 \\ 1\end{array}\right)$-OT or chosen one-out-of-two oblivious transfer we denote the following primitive between a sender $A$ and a receiver $B$. $A$ has two inputs $b_{0}$ and $b_{1}$ and no output, and $B$ has input $c$ and output $y$ such that $y=b_{c}$.

A

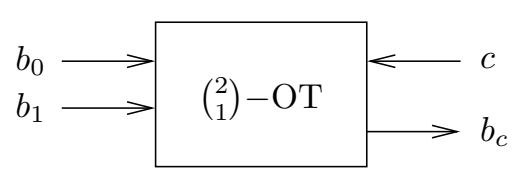

Fig. 1. Chosen one-out-of-two oblivious transfer

For the reversed version of $\left(\begin{array}{l}2 \\ 1\end{array}\right)$ - OT, where $B$ is the sender and $A$ is the receiver, we will write $\left(\begin{array}{l}2 \\ 1\end{array}\right)-\mathrm{TO}$.

This black-box model of oblivious transfer is called the ideal model, and it is how the world is supposed to be: The players have no other way of accessing the box than by the defined inputs and outputs: cheating is impossible. However, in reality such a perfect box does normally not exist. It must be simulated by a protocol. In this real model, the players can cheat in principle by not following the rules. A protocol is called a secure implementation of oblivious transfer if an adversary cannot do anything in the real model that he could not just as well have done in the ideal model. Thus, it must be shown that for any adversary in the real model, there exists an equivalent adversary in the ideal model: he gets the same information and the honest player obtains the same outputs as in the real model; the resulting views are indistinguishable.

We follow the formalism of [16] and [1] (see also [11]) to define when a protocol perfectly securely evaluates a function $f: \mathcal{X} \times \mathcal{Y} \rightarrow \mathcal{U} \times \mathcal{V}$. A protocol is a pair of algorithms $A=\left(A_{1}, A_{2}\right)$ that can interact by two-way message exchange. A pair $\left(\overline{A_{1}}, \overline{A_{2}}\right)$ of algorithms is admissible for protocol $A$ if at least one of the parties is honest, i.e., if $\overline{A_{1}}=A_{1}$ or $\overline{A_{2}}=A_{2}$ holds. (Note that in the case where both parties are cheaters, no security is required.) By $z$, we denote some additional auxiliary input that can potentially be used by both parties. For instance, $z$ could include information about previous executions of the protocol. Note, however, that an honest party never makes use of $z$.

The Ideal Model. In the ideal model, the two parties can make use of a trusted party to calculate the function. The algorithms $\overline{B_{1}}$ and $\overline{B_{2}}$ of the protocol $\bar{B}=\left(\overline{B_{1}}, \overline{B_{2}}\right)$ receive the inputs $x$ and $y$, respectively, and the auxiliary input $z$. They send values $x^{\prime}$ and $y^{\prime}$ to the trusted party, who sends them back the values $u^{\prime}$ and $v^{\prime}$-satisfying $\left(u^{\prime}, v^{\prime}\right)=f\left(x^{\prime}, y^{\prime}\right)$. Finally, $\overline{B_{1}}$ and $\overline{B_{2}}$ output the values $u$ and $v$. The two honest algorithms $B_{1}$ and $B_{2}$ always send $x^{\prime}=x$ and $y^{\prime}=y$ to the trusted party, and always output $u=u^{\prime}$ and $v=v^{\prime}$. Now, if $\bar{B}=\left(\overline{B_{1}}, \overline{B_{2}}\right)$ is 
an admissible pair of algorithms for protocol $B=\left(B_{1}, B_{2}\right)$, the joint execution of $f$ under $\bar{B}$ in the ideal model,

$$
\text { ideal }_{f, \bar{B}(z)}(x, y),
$$

is the resulting output pair, given the inputs $x$ and $y$ and the auxiliary input $z$.

The Real Model. In the real model, the parties have to compute $f$ by a protocol $\Pi=\left(A_{1}, A_{2}\right)$ without the help of a trusted party. Let $\bar{A}=\left(\overline{A_{1}}, \overline{A_{2}}\right)$ be an admissible pair for $A$. Then the joint execution of $\Pi$ under $\bar{A}$ in the real model,

$$
\operatorname{real}_{\Pi, \bar{A}(z)}(x, y) \text {, }
$$

is the resulting output pair, given the inputs $x$ and $y$ and the auxiliary input $z$.

Perfect Security: "Real = Ideal". A protocol $\Pi$ computes a function $f$ perfectly securely if, intuitively speaking, every "real" cheater has an equally powerful counterpart in the ideal model. Definition 2 also applies to reduction protocols from one functionality to another; here, the algorithms are allowed to call an oracle which perfectly implements the given functionality.

Definition 2. A protocol $\Pi$ computes $f$ perfectly securely if for every admissible $\bar{A}=\left(\overline{A_{1}}, \overline{A_{2}}\right)$ there exists an admissible $\bar{B}=\left(\overline{B_{1}}, \overline{B_{2}}\right)$ - as efficient as $\bar{A}^{1}$ and with identical set of honest players - such that for all $x \in \mathcal{X}, x \in \mathcal{Y}$, and $z \in \mathcal{Z}$,

$$
\operatorname{real}_{\Pi, \bar{A}(z)}(x, y) \equiv \operatorname{ideal}_{f, \bar{B}(z)}(x, y)
$$

holds, where $\equiv$ means that the distributions are identical.

\section{Storing and Reversing Oblivious Transfer}

Oblivious transfer protocols rely either on tools borrowed from public-key cryptography [19], [10] or on additional assumptions [3], [6], [20], [4], [23]. In the first case, we have to deal with relatively slow algorithms which may be the bottleneck of the protocol execution. In the second case, one depends on these additional assumptions being present at the time of the execution of the protocol. In both cases it is, therefore, desirable to carry out as much of the computation as possible in advance, and to make the actual execution of oblivious transfer as fast and simple as possible, based on this pre-computation. Actually, almost the entire computation can be done beforehand: Protocols 1 and 2, proposed in [2], show how $\left(\begin{array}{l}2 \\ 1\end{array}\right)$-OT can be transformed into a so-called oblivious key, and vice versa.

An oblivious key is, intuitively speaking, the distribution that arises when $A$ and $B$ choose their inputs at random and execute $\left(\begin{array}{l}2 \\ 1\end{array}\right)$-OT.

Definition 3. By an oblivious key, $\left(\begin{array}{l}2 \\ 1\end{array}\right)-\mathrm{OK}$, we denote the primitive where a sample of two random variables $U=\left(X_{0}, X_{1}\right)$ and $V=(C, Y)$ is given to $A$ and $B$, respectively, where $X_{0}, X_{1}$, and $C$ are independently and uniformly distributed bits, and where $Y=X_{C}$ holds.

\footnotetext{
${ }^{1}$ The running time of $\bar{B}$ must by polynomial in the running time of $\bar{A}$.
} 
Note that $\left(\begin{array}{l}2 \\ 1\end{array}\right)-\mathrm{OK}$ is a key for oblivious transfer in very much the same sense as a shared secret bit is an encryption key in the one-time pad.

Protocol 1. $\left(\begin{array}{l}2 \\ 1\end{array}\right)$-OK from $\left(\begin{array}{l}2 \\ 1\end{array}\right)-\mathrm{OT}$

A $\quad$ B

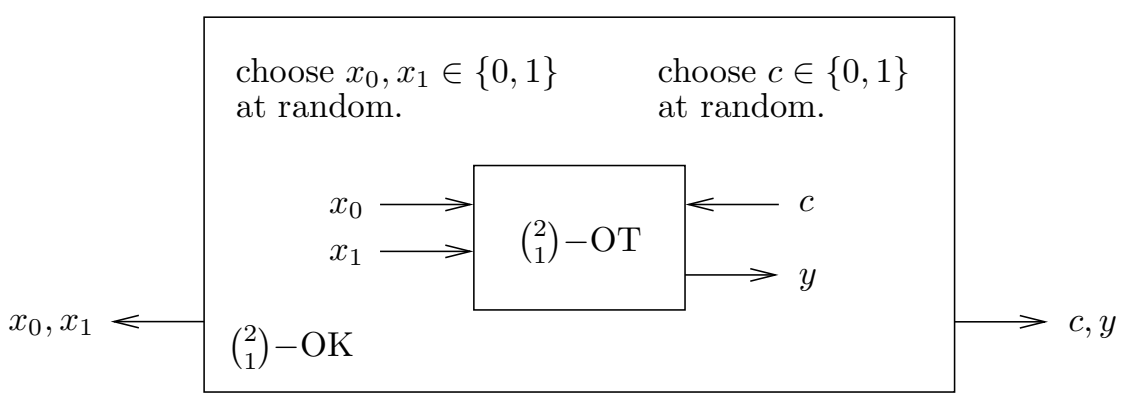

Protocol 2. $\left(\begin{array}{l}2 \\ 1\end{array}\right)$-OT from $\left(\begin{array}{l}2 \\ 1\end{array}\right)-\mathrm{OK}$

A B

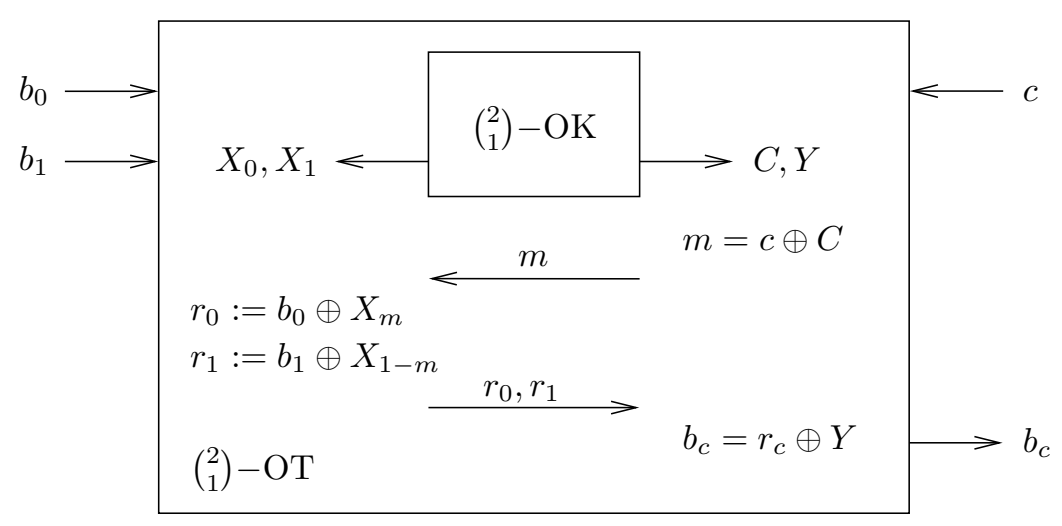

The proofs that Protocols 1 and 2 are perfect single-copy reductions between the primitives $\left(\begin{array}{l}2 \\ 1\end{array}\right)$-OT and $\left(\begin{array}{l}2 \\ 1\end{array}\right)-\mathrm{OK}$ are given in [2] (and straight-forward). Note that both Protocols 1 and 2 work in the honest-but-curious model, whereas their combination is even perfectly secure in the malicious model.

The distribution of $\left(\begin{array}{l}2 \\ 1\end{array}\right)-\mathrm{OK}$ is given and illustrated on the left hand side of Figure 2.

$$
P_{U V}\left(\left(x_{0}, x_{1}\right),(c, y)\right)= \begin{cases}1 / 8 & \text { if } y=x_{c} \\ 0 & \text { otherwise }\end{cases}
$$

When the symbols of $U$ and $V$ are renamed in a suitable way, the distribution corresponds to the one arising when Shannon's so-called "noisy-typewriter channel" [21] is used with random input (see on the right hand side of Figure 2). Obviously, this distribution is symmetric. On the other hand, $\left(\begin{array}{l}2 \\ 1\end{array}\right)-\mathrm{OK}$ 
$U=\left(X_{0}, X_{1}\right) \quad V=(C, Y)$

$(0,0)$

$(1,1)$

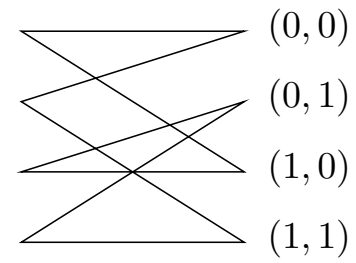

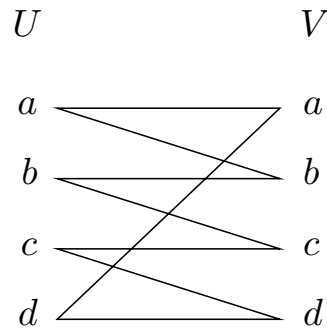

V

Fig. 2. Left hand side: The distribution of $\left(\begin{array}{l}2 \\ 1\end{array}\right)$-OK. (Each edge is a possible combination with probability 1/8.) Right hand side: The distribution arising from the "noisy-typewriter channel." Obviously, the two distributions are equivalent.

is equivalent to $\left(\begin{array}{l}2 \\ 1\end{array}\right)$-OT, which is, hence, symmetric as well: $A$ single instance of $\left(\begin{array}{l}2 \\ 1\end{array}\right)$-TO allows for generating a realization of $\left(\begin{array}{l}2 \\ 1\end{array}\right)$-OT. The reduction is not only single-copy but also perfect, i.e., unconditionally secure without any error. This solves an open problem posed in [9] in a very simple way. Lemma 1 shows how the values in the distribution of a $\left(\begin{array}{l}2 \\ 1\end{array}\right)-\mathrm{OK}$ must be renamed in order for the oblivious key to be reversed.

Lemma 1. Let $X_{0}, X_{1}, C$, and $Y$ be binary random variables and let $(U, V)=$ $\left(\left(X_{0}, X_{1}\right),(C, Y)\right)$ be a $\left(\begin{array}{l}2 \\ 1\end{array}\right)-\mathrm{OK}$. Then $\left(\left(\overline{X_{0}}, \overline{X_{1}}\right),(\bar{C}, \bar{Y})\right):=\left((Y, C \oplus Y),\left(X_{0} \oplus\right.\right.$ $\left.\left.X_{1}, X_{0}\right)\right)$ is a $\left(\begin{array}{l}2 \\ 1\end{array}\right)-\mathrm{OK}$ as well.

Proof. $\bar{Y}=X_{0}=X_{C} \oplus C\left(X_{0} \oplus X_{1}\right)=Y \oplus C\left(X_{0} \oplus X_{1}\right)=\overline{X_{0}} \oplus\left(\overline{X_{0}} \oplus \overline{X_{1}}\right) \bar{C}=\bar{X}_{\bar{C}}$.

A formal proof of the security of this transformation is omitted here. Intuitively, the privacy of both players is preserved since the ignorance of one player about the XOR of $X_{0}$ and $X_{1}$ is transformed into the ignorance of $C$, and vice versa.

\section{Optimally Reversing Oblivious Transfer}

The protocol outlined in the end of Section 3 requires three bits of additional communication. We present an even simpler protocol, Protocol 3, using only one bit of additional communication from $A$ to $B$; this is optimal.

Theorem 1. Protocol 3 perfectly securely reduces $\left(\begin{array}{l}2 \\ 1\end{array}\right)$-OT to one realization of $\left(\begin{array}{l}2 \\ 1\end{array}\right)-\mathrm{TO}$.

Proof. Let first both parties be honest, i.e., $\bar{A}=\left(A_{1}, A_{2}\right)$ in Protocol 3. Then we have, for all $\left(b_{0}, b_{1}\right) \in\{0,1\}^{2}, c \in\{0,1\}$, and $z \in \mathcal{Z}$,

$$
\begin{aligned}
\operatorname{real}_{3, \bar{A}(z)}\left(\left(b_{0}, b_{1}\right), c\right) & =\left(\perp, r \oplus\left(b_{0} \oplus a\right)\right) \\
& =\left(\perp, b_{0} \oplus\left(b_{0} \oplus b_{1}\right) c\right) \\
& =\left(\perp, b_{c}\right) \\
& =\text { ideal }_{\left(\begin{array}{l}
2 \\
1
\end{array}\right)-\mathrm{OT}, B(z)}\left(\left(b_{0}, b_{1}\right), c\right) .
\end{aligned}
$$


Protocol 3. $\left(\begin{array}{l}2 \\ 1\end{array}\right)$-OT from $\left(\begin{array}{l}2 \\ 1\end{array}\right)-\mathrm{TO}$

A

B

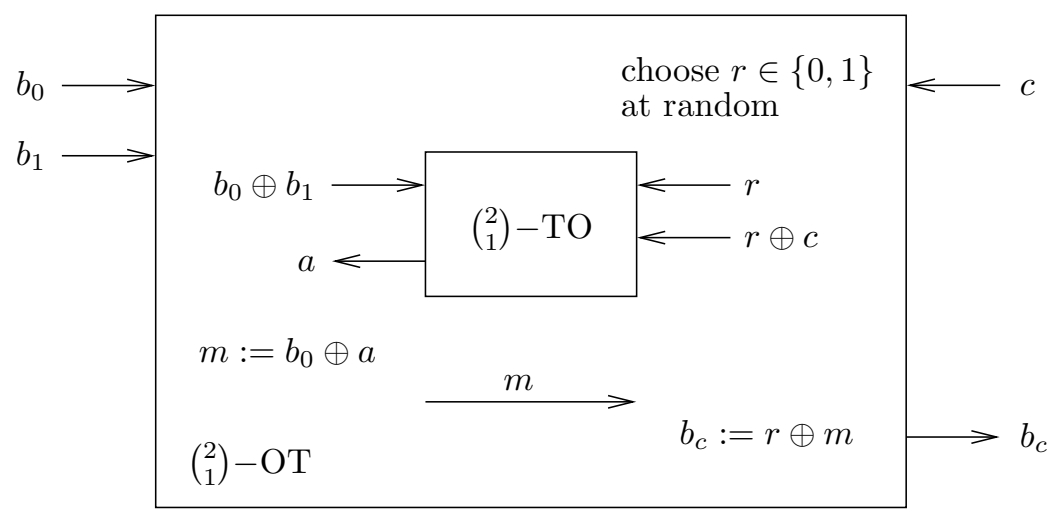

Let now the first party be honest, i.e., $\bar{A}=\left(A_{1}, \overline{A_{2}}\right)$. In the real model, $\overline{A_{2}}$ receives $(c, z)$ and sends $\left(a_{0}, a_{1}\right)=a(c, z)$ to $\left(\begin{array}{l}2 \\ 1\end{array}\right)-$ TO. Then he receives $m=b_{0} \oplus a_{b_{0} \oplus b_{1}}$, and outputs $v\left(c, z, a_{0}, a_{1}, m\right)$. Let the adversary $\overline{B_{2}}$ in the ideal model be defined as follows: On inputs $(c, z)$, he sends $(c, z)$ to $\overline{A_{2}}$, and gets $\left(\overline{a_{0}}, \overline{a_{1}}\right)=a(c, z)$ back. He sends $\bar{c}:=\overline{a_{0}} \oplus \overline{a_{1}}$ to $\left(\begin{array}{l}2 \\ 1\end{array}\right)-\mathrm{OT}$ and gets $b_{\bar{c}}$ back. Then he sends $\bar{m}:=b_{\bar{c}} \oplus \overline{a_{0}}$ to $\overline{A_{2}}$, gets $\bar{v}=v\left(c, z, \overline{a_{0}}, \overline{a_{1}}, \bar{m}\right)$ back and outputs $\bar{v}$.

Since

$$
\bar{m}=\overline{a_{0}} \oplus b_{\bar{c}}=\overline{a_{0}} \oplus b_{\overline{a_{0}} \oplus \overline{a_{1}}}=b_{0} \oplus \overline{a_{0}} \oplus\left(b_{0} \oplus b_{1}\right)\left(\overline{a_{0}} \oplus \overline{a_{1}}\right)=b_{0} \oplus \overline{a_{b_{0} \oplus b_{1}}},
$$

we have, for all $\left(b_{0}, b_{1}\right) \in\{0,1\}^{2}, c \in\{0,1\}$, and $z \in \mathcal{Z}$,

$$
\begin{aligned}
\operatorname{real}_{3, \bar{A}(z)}\left(\left(b_{0}, b_{1}\right), c\right) & =\left(\perp, v\left(c, z, a_{0}, a_{1}, m\right)\right) \\
& =\left(\perp, v\left(c, z, a_{0}, a_{1}, b_{0} \oplus a_{b_{0} \oplus b_{1}}\right)\right) \\
& \equiv\left(\perp, v\left(c, z, \overline{a_{0}}, \overline{a_{1}}, b_{0} \oplus \overline{a_{b_{0} \oplus b_{1}}}\right)\right) \\
& =\left(\perp, v\left(c, z, \overline{a_{0}}, \overline{a_{1}}, \bar{m}\right)\right) \\
& =\operatorname{ideal}_{\left(\begin{array}{l}
2 \\
1
\end{array}\right)-\mathrm{OT},\left(B_{1}, \overline{B_{2}}\right)(z)}\left(\left(b_{0}, b_{1}\right), c\right) .
\end{aligned}
$$

Assume now that the second party is honest, i.e., $\bar{A}=\left(\overline{A_{1}}, A_{2}\right)$. In the real model, $\overline{A_{1}}$ receives $\left(\left(b_{0}, b_{1}\right), z\right)$ and sends $d=d\left(\left(b_{0}, b_{1}\right), z\right)$ to $\left(\begin{array}{l}2 \\ 1\end{array}\right)-\mathrm{TO}$, which returns $l=r \oplus d c$. Then, he sends $m=m\left(\left(b_{0}, b_{1}\right), z, l\right)$ to $A_{2}$ and outputs $u\left(\left(b_{0}, b_{1}\right), z, d, l, m\right)$. Let the adversary $\overline{B_{1}}$ in the ideal model be defined as follows: On inputs $\left(\left(b_{0}, b_{1}\right), z\right), \overline{B_{1}}$ sends $\left(\left(b_{0}, b_{1}\right), z\right)$ to $\overline{A_{1}}$ and gets $\bar{d}=d\left(\left(b_{0}, b_{1}\right), z\right)$ back. He chooses $\bar{l}$ uniformly at random and sends it to $\overline{A_{1}}$, who sends $\bar{m}=m\left(\left(b_{0}, b_{1}\right), z, \bar{l}\right)$ and $\bar{u}=u\left(\left(b_{0}, b_{1}\right), z, \bar{d}, \bar{l}, \bar{m}\right)$ back. He sends $(\bar{l} \oplus \bar{m}, \bar{l} \oplus \bar{m} \oplus d)$ to $\left(\begin{array}{l}2 \\ 1\end{array}\right)-\mathrm{OT}$ and outputs $\bar{u}$.

The honest player will output $\bar{l} \oplus \bar{m} \oplus c \bar{d}$. Since $l=r \oplus d c$ and $r$ is uniform and independent of everything else, $l$ is uniform and independent as well, which 
means that it has the same joint distribution as $\bar{l}$ with everything else. Therefore we have, for all $\left(b_{0}, b_{1}\right) \in\{0,1\}^{2}, c \in\{0,1\}$, and $z \in \mathcal{Z}$,

$$
\begin{aligned}
\operatorname{real}_{3, \bar{A}(z)}\left(\left(b_{0}, b_{1}\right), c\right) & =\left(u\left(\left(b_{0}, b_{1}\right), z, d, l, m\right), r \oplus m\right) \\
& =\left(u\left(\left(b_{0}, b_{1}\right), z, d, l, m, l \oplus d c \oplus m\right)\right. \\
& \equiv\left(u\left(\left(b_{0}, b_{1}\right), z, \bar{d}, \bar{l}, \bar{m}, \bar{l} \oplus \bar{d} c \oplus \bar{m}\right)\right. \\
& =\operatorname{ideal}_{\left(\begin{array}{l}
2 \\
1
\end{array}\right)-\mathrm{OT}, \bar{B}(z)}\left(\left(b_{0}, b_{1}\right), c\right) .
\end{aligned}
$$

Obviously, the simulated adversary is as efficient as the real adversary.

Our protocol is optimal: First of all, since it is impossible to construct unconditionally secure oblivious transfer from scratch, using a single instance of $\left(\begin{array}{l}2 \\ 1\end{array}\right)$ - TO is optimal. Since $\left(\begin{array}{l}2 \\ 1\end{array}\right)$ - TO does not allow any communication from Bob to Alice, but $\left(\begin{array}{l}2 \\ 1\end{array}\right)$-OT does allow one bit of communication, any protocol must communicate at least one bit. Furthermore, there cannot exist a protocol where Bob does not use any randomness, because then his inputs to $\left(\begin{array}{l}2 \\ 1\end{array}\right)$ - TO would be deterministic functions of $c$. These functions could not both be constant, since then the output of $\left(\begin{array}{l}2 \\ 1\end{array}\right)$-TO would not depend on $c$ and be useless, and therefore no oblivious transfer would be possible. But if the functions are not constant, $A$ is able to obtain information about $c$.

\section{Oblivious Linear-Function Evaluation}

In contrast to $\left(\begin{array}{l}2 \\ 1\end{array}\right)-\mathrm{OT}$, all the other forms of oblivious transfer cannot be reversed without loss, i.e., in the perfect single-copy sense of Sections 3 and 4 . This can easily be seen from the monotones, defined in [24]: A primitive can only be reversed without loss if

$$
H(Y \searrow X \mid X)=H(X \searrow Y \mid Y),
$$

and $\left(\begin{array}{l}2 \\ 1\end{array}\right)$-OT is the only example of $\left(\begin{array}{l}n \\ k\end{array}\right)-\mathrm{OT}^{l}$ having this property.

In this section, we present another natural generalization of oblivious transfer to strings that can be reversed perfectly: oblivious linear-function evaluation over $G F(q)$ or $G F(q)$-OLFE for short. Roughly speaking, the sender's input is a linear function $f: x \mapsto y=a_{0}+a_{1} x$, where $a_{0}, a_{1}, x, y \in G F(q)$, and the receiver's input is an argument $x \in G F(q)$ for which he then learns the evaluation of the function, $y=f(x)$ (see Figure 3). $G F(q)-$ OLFE is a special case of oblivious polynomial evaluation [17]. It can easily be verified that $G F(2)-O L F E$ is equivalent to $\left(\begin{array}{l}2 \\ 1\end{array}\right)-\mathrm{OT}$. Furthermore, [20] shows that with one instance of $G F(q)-\mathrm{OLFE}$ a very simple commitment scheme can be implemented, which allows to commit to a value $x \in G F(q)$. The scheme is perfectly hiding and $1 / q$-binding.

The protocols of Sections 3 and 4 generalize to $G F(q)$-OLFE in a straightforward way: $G F(q)-\mathrm{OLFE}$ is, as oblivious transfer, equivalent to a non-interactive key - and can, therefore, be stored in the same sense. Moreover, this key is, as $\left(\begin{array}{l}2 \\ 1\end{array}\right)-\mathrm{OK}$, symmetric. Hence, $G F(q)-\mathrm{OLFE}$ from $A$ to $B$ can be reduced to 
A

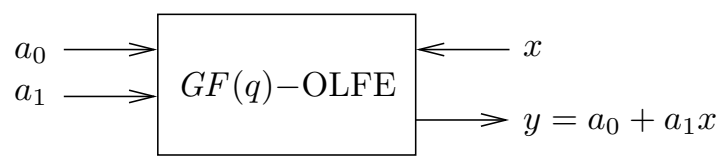

Fig. 3. Oblivious linear-function evaluation over $G F(q)$

$G F(q)$-OLFE from $B$ to $A-G F(q)$-EFLO for short - in a perfect and singlecopy sense. Protocol 4 is, in addition, optimal with respect to the required communication.

Protocol 4: $G F(q)-\mathrm{OLFE}$ from $G F(q)-\mathrm{EFLO}$

A

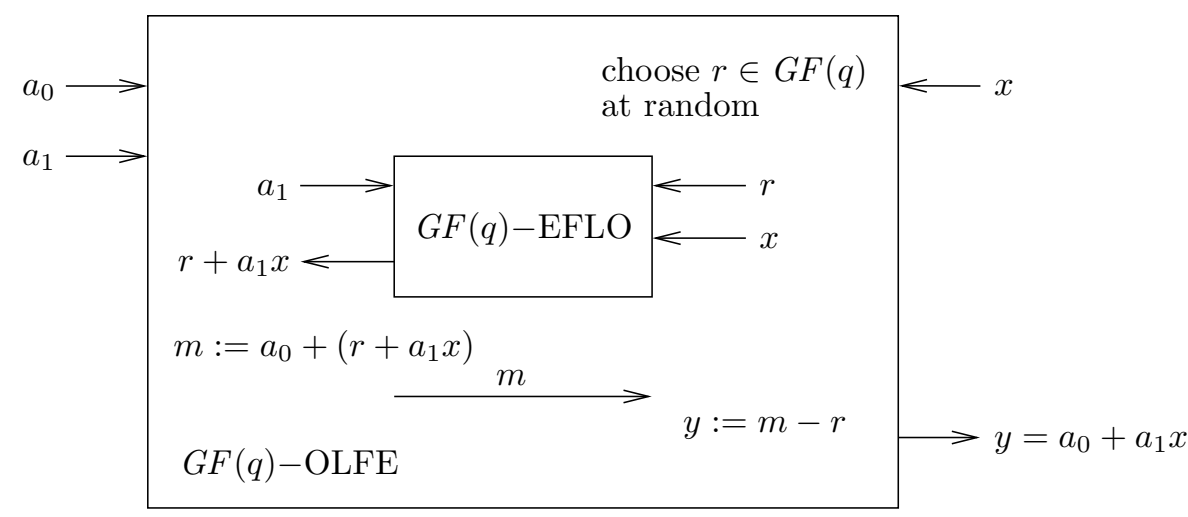

\section{Concluding Remarks}

We have shown that chosen one-out-of-two bit oblivious transfer can be optimally reversed very easily. Furthermore, we have have presented a more general primitive with the same property: oblivious linear-function evaluation.

\section{Acknowledgments}

This work was carried out while both authors were with Université de Montréal, Canada. This research was supported by Canada's NSERC, Québec's FQRNT, and Switzerland's SNF.

\section{References}

1. D. Beaver, Foundations of Secure Interactive Computing, Advances in Cryptology -Proceedings of CRYPTO '91, LNCS, Vol. 576, pp. 377-391, Springer-Verlag, 1992.

2. D. Beaver, Precomputing oblivious transfer, Advances in Cryptology-Proceedings of CRYPTO '95, LNCS, Vol. 963, pp. 97-109, Springer-Verlag, 1995. 
3. C. H. Bennett, G. Brassard, C. Crépeau, and H. Skubiszewska, Practical quantum oblivious transfer, Advances in Cryptology - Proceedings of EUROCRYPT '91, LNCS, Vol. 576, pp. 351-366, Springer-Verlag, 1992.

4. G. Brassard, C. Crépeau, and S. Wolf, Oblivious transfers and privacy amplification, Journal of Cryptology, Vol. 16, No. 4, pp. 219-237, 2003.

5. C. Crépeau, Correct and private reductions among oblivious transfers, $\mathrm{Ph}$. D. Thesis, Massachusetts Institute of Technology, 1990.

6. C. Crépeau, Efficient cryptographic protocols based on noisy channels, Advances in Cryptology-Proceedings of CRYPTO '97, LNCS, Vol. 1233, pp. 306-317, SpringerVerlag, 1997.

7. C. Crépeau and J. Kilian, Achieving oblivious transfer using weakened security assumptions, Proceedings of the $28^{\text {th }}$ Symposium on Foundations of Computer Science (FOCS '88), pp. 42-52, IEEE, 1988.

8. C. Crépeau, K. Morozov, and S. Wolf, Efficient unconditional oblivious transfer from almost any noisy channel, Proceedings of Fourth Conference on Security in Communication Networks (SCN) '04, LNCS, Springer-Verlag, 2004.

9. C. Crépeau and M. Sántha, On the reversibility of oblivious transfer, Advances in Cryptology-Proceedings of EUROCRYPT '91, LNCS, Vol. 547, pp. 106-113, Springer-Verlag, 1991.

10. S. Even, O. Goldreich, and A. Lempel, A randomized protocol for signing contracts, Communications of the ACM, Vol. 28, No. 6, pp. 637-647, 1985.

11. O. Goldreich, Foundations of Cryptography, Volume II: Basic Applications, Cambridge University Press, 2004.

12. H. Imai, J. Müller-Quade, A. Nascimento, and A. Winter, Rates for bit commitment and coin tossing from noisy correlation, Proceedings of the IEEE International Symposium on Information Theory (ISIT) '04, IEEE, 2004.

13. H. Imai, A. Nascimento, and A. Winter, Oblivious transfer from any genuine noise, unpublished manuscript, 2004.

14. J. Kilian, Founding cryptography on oblivious transfer, Proceedings of the Twentieth Annual ACM Symposium on Theory of Computing (STOC '88), pp. 20-31, 1988 .

15. D. Mayers, Unconditionally secure quantum bit commitment is impossible, Phys. Rev. Lett., Vol. 78, pp. 3414-3417, 1997.

16. S. Micali and P. Rogaway, Secure computation, Advances in Cryptology-Proceedings of CRYPTO '91, LNCS, Vol. 576, pp. 392-404, Springer-Verlag, 1992.

17. M. Naor and B. Pinkas. Oblivious transfer and polynomial evaluation, Proceedings of the Thirty-First Annual ACM Symposium on Theory of Computing (STOC '99), pp. 245-354, 1999.

18. R. Ostrovsky, R. Venkatesan, and M. Yung, Fair games against an all-powerful adversary, AMS DIMACS Series in Discrete Mathematics and Theoretical Computer Science, Vol. 13, pp. 155-169, 1990.

19. M. Rabin, How to exchange secrets by oblivious transfer, Technical Report TR-81, Harvard Aiken Computation Laboratory, 1981.

20. R. L. Rivest, Unconditionally secure commitment and oblivious transfer schemes using private channels and a trusted initializer, unpublished manuscript, 1999.

21. C. E. Shannon, A mathematical theory of communication, Bell System Technical Journal, Vol. 27, pp. 379-423, 623-656, 1948.

22. A. Winter, A. Nascimento, and H. Imai, Commitment capacity of discrete memoryless channels, Cryptography and Coding, LNCS, Vol. 2898, pp. 35-51, SpringerVerlag, 2003. 
23. S. Wolf and J. Wullschleger, Zero-error information and applications in cryptography, Information Theory Workshop (ITW) '04, IEEE, 2004.

24. S. Wolf and J. Wullschleger. New monotones and lower bounds in unconditional two-party computation. In Advances in Cryptology-Proceedings of CRYPTO '05, LNCS, Vol. 3621, pp. 467-477, Springer-Verlag, 2005.

25. A. D. Wyner, The wire-tap channel, Bell System Technical Journal, Vol. 54, No. 8, pp. 1355-1387, 1975.

26. R. W. Yeung, A new outlook on Shannon's information measures, IEEE Transactions on Information Theory, Vol. 37, No. 3, pp. 466-474, 1991. 